\title{
Design of LED Light Music Rhythm Based on Virtual Instrument
}

\author{
Yuan Wang ${ }^{1,2 *}$ and Liping Huang ${ }^{3}$ \\ ${ }^{1}$ Institute of Science and Technology, University of Sanya, Sanya, Hainan, China \\ ${ }^{2}$ Institute of Maritime Communication, University of Sanya, Sanya, Hainan, China \\ ${ }^{3}$ Sanya No.1 Middle School, Sanya, Hainan, China \\ ${ }^{*}$ Corresponding author
}

\begin{abstract}
The article proposes a design scheme of LED light music rhythm based on virtual instrument, studying the audio signal collection, processing and application. Instead of a general data collection card, using computer sound card as hardware acquisition audio signal and transmit it to the computer. On the LabVIEW software platform, based on signal processing function, audio playback and signal processing system is designed, which analysis and process the audio signal at different frequencies of input signal strength to generate the output signal associated with the frequency and intensity of the input audio signal, so that realizes the LabVIEW virtual LED matrix in the program and the actual LED matrix real-time synchronously to flicker with the rhythm of the music.

The design of the audio signal acquisition and processing system is provided with good human-machine interface, strong real-time performance and convenient operation. It can be extended its function according to the needs of user. With broad application prospect, it can be applied to a variety of audio signal collection and processing fields such as speech recognition, environmental noise monitoring, etc.
\end{abstract}

Keywords-virtual instrument; sound card; data acquisition; audio signal processing; LED Matrix

\section{INTRODUCTION}

The virtual instrument is a product of a combination of modern computer technology and instrument technology. It was produced in the late 1980s, and it has a major breakthrough in concept and function compared to traditional instruments. The virtual instrument makes full use of the computer's intelligent functions such as computing, storage, calculation, playback display, and file management [1]. At the same time, the specialized functions and panel controls of the traditional instrument are softwareized to combine it with the computer to form a fully functional and conventional hardware. The same instrument, but also fully enjoy the computer hardware and software resources of the new virtual instrument system. The emergence of virtual instruments completely changed the traditional concept of instruments. Signal processing is an important part of engineering testing. This is also a link that can fully reflect the great advantages of virtual instrument technology in engineering testing. Dynamic signal processing includes digital filtering, FFT spectrum, correlation analysis, coherent analysis, frequency response and signal generation, special function analysis, and acoustic analysis [2].
This article aims to use virtual instrument development software LabVIEW, design a LED music rhythm lamp design based on virtual instrument technology, use the sound card to acquire the signal, transmit it to the computer, process the signal and analyze the frequency in the LabVIEW program, and generate the frequency of the input signal The output signal, to control the LED light flashes with the music playing rhythm.

\section{LABVIEW SOFTWARE INTRODUCTION}

LabVIEW is a program development environment developed by National Instruments (NI) Corporation. It is similar to the $\mathrm{C}$ and BASIC development environments. However, LabVIEW differs significantly from other computer languages in that other computer languages use a text-based language to generate code. While LabVIEW uses a graphical editing language $\mathrm{G}$ to write programs, the resulting program is in the form of a block diagram. LabVIEW provides many controls that look similar to traditional instruments (such as oscilloscope, multimeter) and can be easily used to create a designed user interface. The front panel is the LabVIEW user interface. It contains icons and wires to programmatically control the objects on the front panel. Graphical source code, also known as $\mathrm{G}$ code [3].

LabVIEW has a wide range of applications and has the greatest advantages in areas such as signal testing, measurement, and automation because LabVIEW provides a large number of tools and calling functions for data acquisition, processing, analysis, display, and storage. Users can complete a complete set of automated test and measurement system from instrument connection, data acquisition to analysis, display and storage in a short time. LabVIEW's flexibility, modularity, and ease of programming make him popular in the labs of universities. Compared with other simulation softwares, its powerful combination with hardware or even "embedded" hardware capabilities makes it easier to implement on hardware, making it easier to verify the accuracy, effectiveness, and practicality of the designed algorithms and systems. At present, it is widely used in various fields such as automotive, communications, aviation, semiconductor, electronic design and production, process control and biomedicine [4].

\section{SYSTEM DESIGN}

Acquire and process audio signal data through computer's sound card and LabVIEW. The program written by LabVIEW 
software to control the reading of music files, using the PC's own sound card to acquire signals and using LabVIEW's own sound card program to set the playing music files, so as to achieve music playback, the LED light group flashes, the audio is much brighter and the audio is less flickering [5].

Software design includes music player module, audio signal acquisition and processing module and LED display module, which is realized through LabVIEW programming. The music playing module is equivalent to a simple MP3 player system. First, the music file is read, and then the music is played by configuring the sound output, starting the sound output, writing the sound output, stopping the sound output, and clearing the sound output. The sound card acquisition module collects the audio analog signal output by the music player module, and then obtains the amplitude of different frequencies through filtering and amplification, thereby controlling the LED display module and achieving the flashing of the LED accompanying the music [6].

The hardware design is mainly filter module, LED display module. Obtain the audio analog output signal through the DAQ data acquisition card, and then pass through different filter modules to obtain the output signals of different frequency ranges, and control the LED rhythm of each channel to blink.

The main function of the system is to click and play the selected audio file. The sound card collects the audio signal, passes through different bandwidths of the virtual band-pass filter and the output amplitude level vi, and then calls the front panel Boolean of the algorithm sub vi control software. With the music playing, the Boolean lights arranged in a column flash. At the same time, the audio signal is output through a DAQ data acquisition card, and a band-pass filter that allows different frequencies to pass through is used. The integrated drive circuit achieves the on and off of the LED lamp, thereby realizing the arrangement of the LED lamp and software along with the music playback, hardware arrangement. Boolean lights flash at the same time. The system structure is shown in the Figure 1.

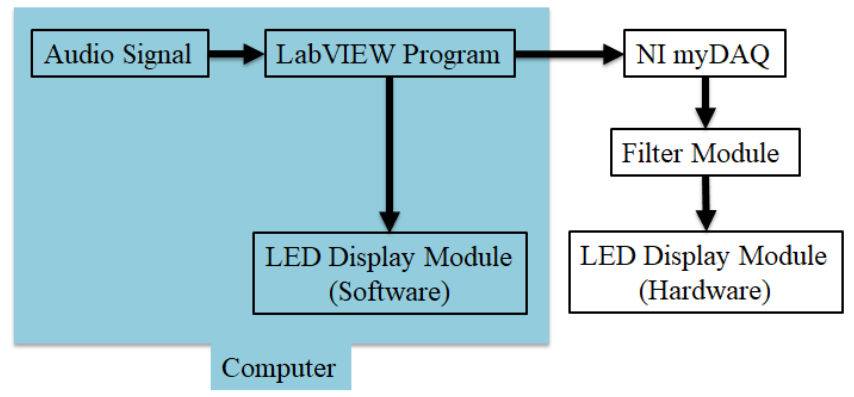

FIGURE I. THE SYSTEM STRUCTURE

\section{DESIGN OF SOFTWARE PROGRAMS}

The software design part mainly includes the audio file playing module, the audio signal acquisition processing module and the LED display module, which is realized through LabVIEW programming. Software design flow chart is shown in the Figure 2.

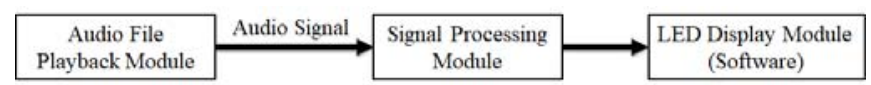

FIGURE II. SOFTWARE DESIGN FLOW CHART

\section{A. Audio File Playback Module}

The audio file playback module is equivalent to a simple MP3 player. Its function includes the selection of music files (format *.wav), volume adjustment, start playback, stop and exit, orange square The display of the waveform melody of the audio signal, the interface is simple and friendly. The design of the audio playback module includes the entire process of data acquisition. The acquisition process is mainly divided into three steps: initializing/configuring the sound card, sampling, and releasing the sound card.

1) Initialize the sound card: Before performing music playback data acquisition, first set the sound card parameters, such as device ID, sampling mode (continuous sampling / limited sampling), number of samples per channel, sampling rate, number of channels (single channel dual channel) and bit rate Wait. LabVIEW provides a series of functions related to sound cards: sound output operation functions, sound input operation functions, and file operation functions. We use the "Sound Input Configure" function (the node diagram shown in the Figure 3) to configure the sound card device parameters that are used to obtain the data and send it to the buffer.

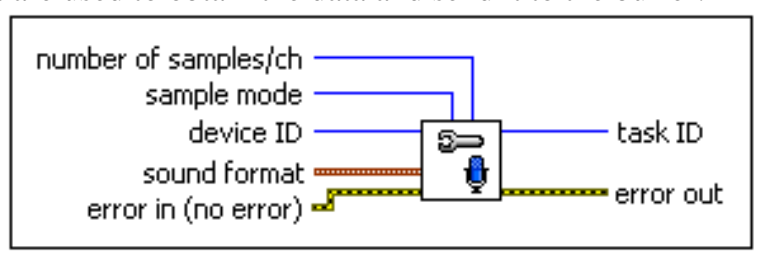

FIGURE III. "SOUND INPUT CONFIGURE" FUNCTION

2) Sampling: According to the parameter settings, after setting each parameter into the loop structure, use the "read sound" function to read the acquisition data signal from the input device and display it through the graphic display. Press the "Stop" button to pause the signal and stop sampling. This module will provide real-time acquisition of signal data for audio signal analysis.

3) Release sound card: With the "sound input clear" function, stop the sound signal acquisition, clear the buffer, return to the default state, and release the task-related resources.

\section{B. Audio Signal Processing Module}

Acquire the audio signal data collected by the sound card through the "read sound input" function and pass it through the band-pass filter with different bandwidths. This design uses six band-pass filters respectively, with frequency ranges of $50 \sim 500 \mathrm{~Hz}, \quad 500 \sim 1000 \mathrm{~Hz}, \quad 1000 \sim 2000 \mathrm{~Hz}, \quad 2000 \sim 3000 \mathrm{~Hz}$, $3000 \sim 4000 \mathrm{~Hz}, 4000 \sim 5000 \mathrm{~Hz}$, one of which is shown in the Figure 4. Obtain the audio signal of different frequency bands, and then output the real-time amplitude array according to the requirement of the amplitude level function VI, and then use the index array function VI to obtain the amplitude level 
elements output in real time, which provides the conditions for the subsequent realization of the corresponding functions. LabVIEW provides a number of function analysis and processing VIs, which brings a lot of convenience to the design.

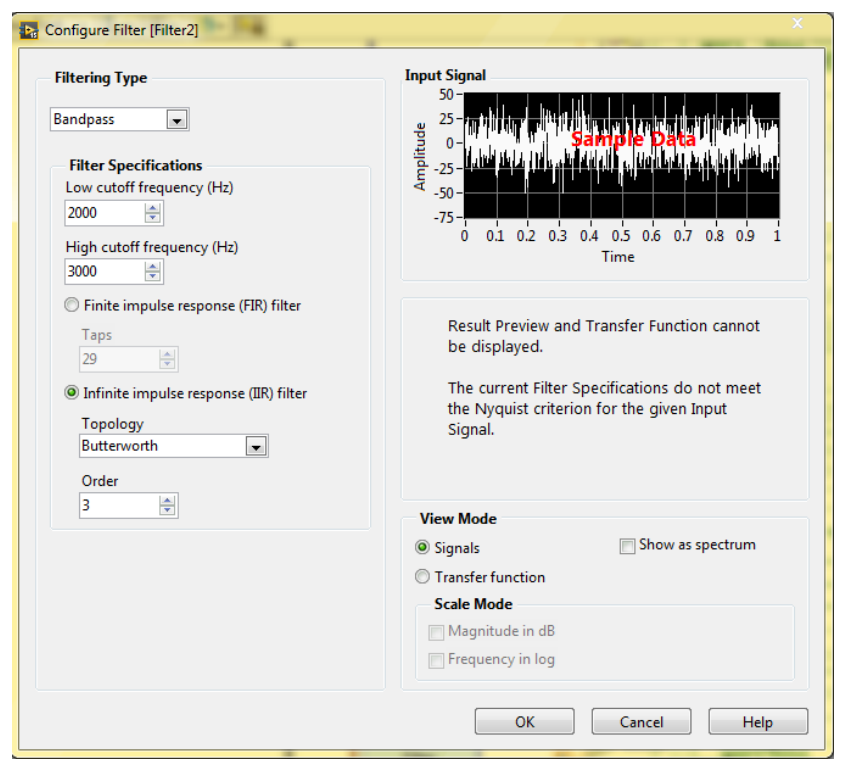

FIGURE IV. INTERFACE OF BAND-PASS FILTER CONFIGURATION

\section{LED Display Module}

According to the conditions provided by the signal processing module, the size of the elements of the real-time level is obtained and amplified, and then the design subVI is invoked to control the rhythm of the LED lamp. When the level amplitude is $0<\mathrm{X}<0.5 \mathrm{~V}$, Boolean1 lights up; when $0.5<\mathrm{X}<1.5 \mathrm{~V}$, Boolean1 and Boolean2 light up simultaneously; when $1.5<\mathrm{X}<2 \mathrm{~V}$, Boolean1, Boolean2, and Boolean3 light up at the same time; When $2<\mathrm{X}<3 \mathrm{~V}$, Boolean1, Boolean2, Boolean3, and Boolean4 light up at the same time, and so on. When the level amplitude $\mathrm{X}>8 \mathrm{~V}$, all Booleans light up. With the music playing, Boolean lights will flash with the audio signal rhythm, audio amplitude is high, Boolean is much brighter, audio amplitude is low, Boolean is bright. In the front panel, Boolean is arranged in the order of the lit amplitude of the audio. In order to make the interface more beautiful, Boolean is arranged in 10 rows and 11 columns and its light color is changed. The display effect is shown in Figure 5.

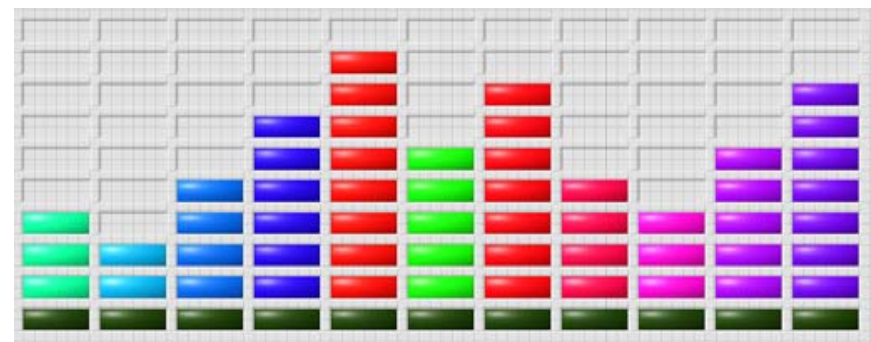

FIGURE V. LED DISPLAY MODULE

\section{HARDWARE CIRCUIT DESIGN}

Connect the computer through the NI myDAQ data acquisition card to collect real-time audio signals, and then use their own digital-analog conversion function to generate audio analog signals to the designed hardware circuit to control the hardware LED matrix real-time synchronous rhythm flashing. The overall circuit diagram of the hardware circuit is shown in Figure 6, and the circuit diagram simulated in the circuit simulation software Protues is shown in Figure 7. The display is designed using integrated operational amplifiers and dedicated integrated circuits. The circuit is simple, stable, easy to manufacture, and easy to use. The display can display the instantaneous level of each frequency point with five dynamic light bars at six frequency points (including a certain bandwidth) at $112 \mathrm{~Hz}, 503 \mathrm{~Hz}, 1591 \mathrm{~Hz}, 2250 \mathrm{~Hz}, 3319 \mathrm{~Hz}$ and $4594 \mathrm{~Hz}$ at the same time.

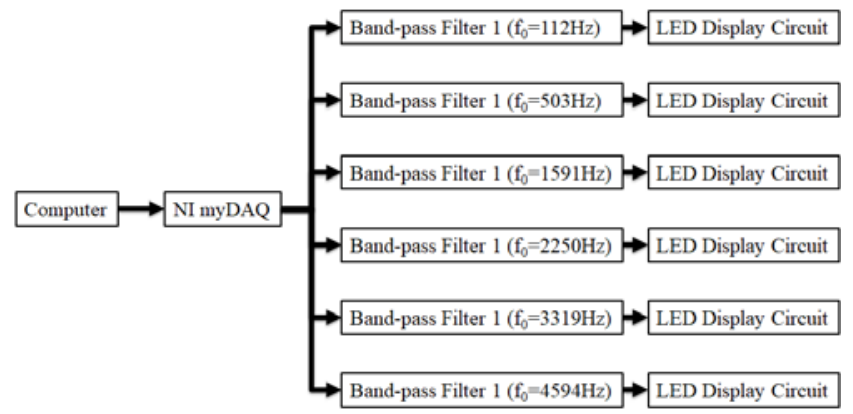

FIGURE VI. OVERALL STRUCTURE DIAGRAM OF HARDWARE CIRCUIT

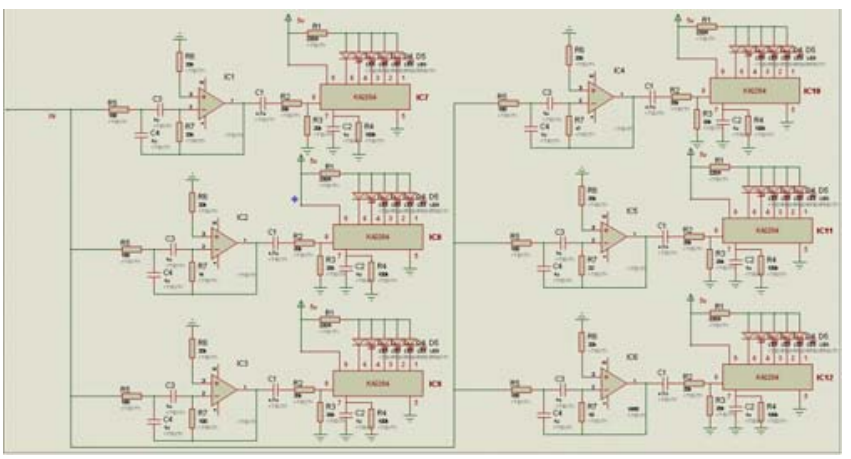

FIGURE VII. SIMULATION CIRCUIT DIAGRAM

The overall working principle: LabVIEW software on the computer to play audio files, DAQ data acquisition card to collect audio signals, and then simultaneously transmitted to the six active band-pass filter, each band-pass filter allows only the signal of a specific frequency range, and then The LED rhythm is controlled by the level drive IC.

\section{A. Active Band-pass Filter}

The design of the hardware is similar to that of the software. Six active band-pass filters are designed. The band-pass filter allows only signals with frequencies in the passband to pass. Signals above the upper cutoff frequency and below the lower cutoff frequency are blocked. The designed filter actual circuit diagram is shown in Figure 8. 


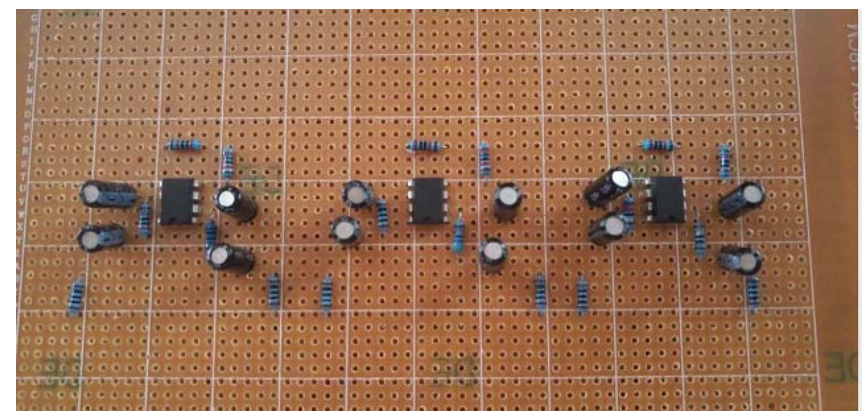

FIGURE VIII. FILTER ACTUAL CIRCUIT DIAGRAM

\section{B. LED Display Module}

The hardware LED display module is mainly composed of a driver IC circuit and an LED display matrix. The magnitude of the signal amplitude level is converted to the level of the LED rhythm beam, requiring the use of a voltage comparator. The voltage comparator using an integrated op amp is shown in Figure 9. The reference voltage $\mathrm{Vr}$ is connected to the noninverting input of the integrated op amp IC and the input signal $\mathrm{Vi}$ is connected to the inverting input of the IC. When the input signal $\mathrm{Vi}$ is less than the reference voltage $\mathrm{Vr}$, the output Vo is high and the light-emitting diodes are off. When the input signal Vi is greater than the reference voltage Vr, the output Vo is low and the light-emitting diodes are on. In order to make the display more rhythmic and beautiful, 11 LEDs and 5 LEDs are arranged on the structure to form an LED matrix.

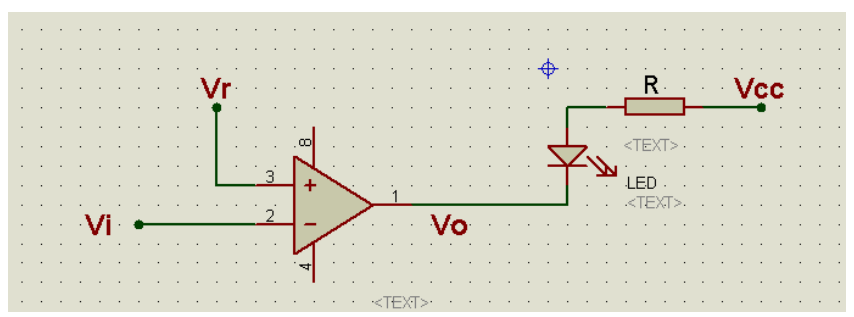

FIGURE IX. VOLTAGE COMPARATOR CIRCUIT

\section{CONCLUSION}

This article proposes a scheme of LED music rhythm lamp design based on virtual instruments, and studies the collection, processing and application of audio signals. A computer sound card is used as the hardware to collect the audio signal instead of the normal data acquisition card and transmitted to the computer. Based on the signal processing function, the audio file playback and signal processing system is designed on the LabVIEW software platform, and the input audio signal is divided into different frequency bands for signal strength. The analysis and processing to generate the output signal related to the frequency and intensity of the input audio signal enables the virtual LED matrix and the actual LED matrix in the LabVIEW program to blink in synchrony with the rhythm of the music in real time. The result of the system operation is shown in Figure 10.

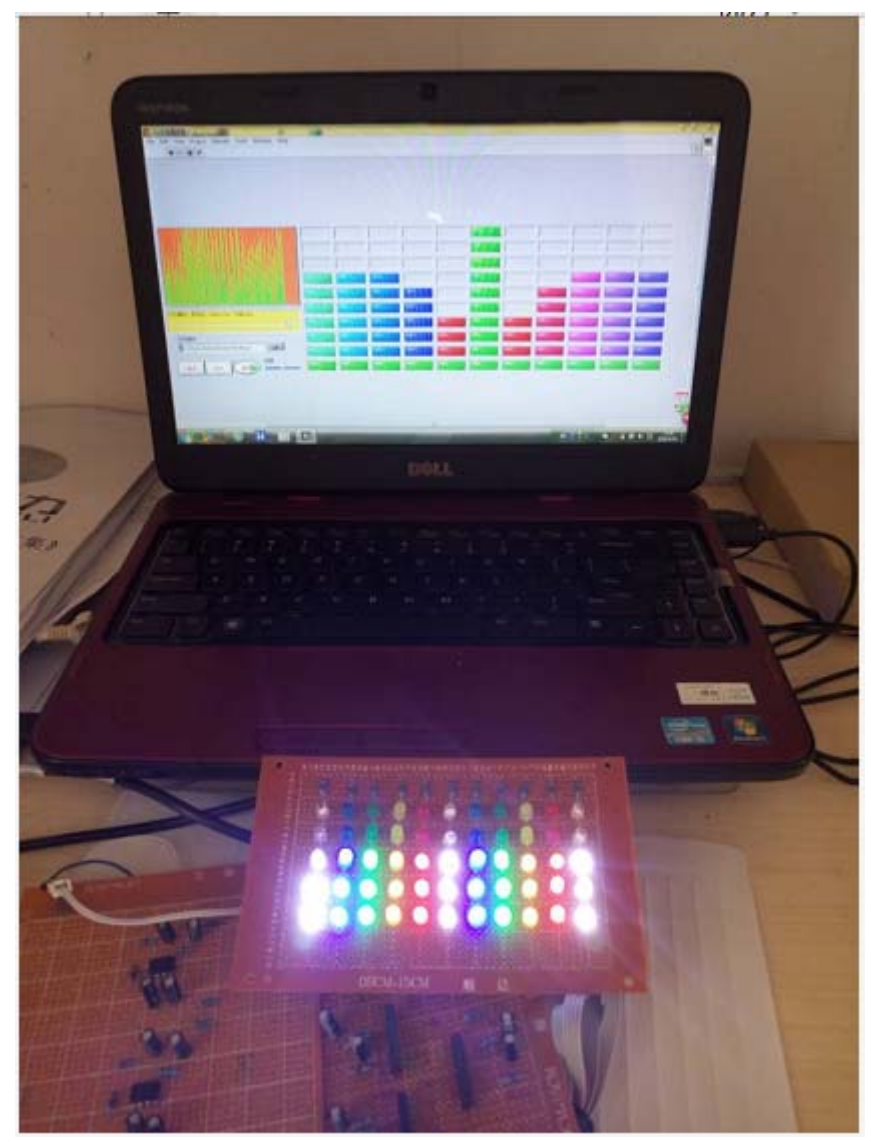

FIGURE X. THE RESULT OF THE SYSTEM

\section{ACKNOWLEDGMENT}

Y. W. thanks the Educational Research Fund of Hainan Province Education Department (Grant No. Hnjg2016ZD-20), the National Student Innovation and Entrepreneurship Training Program (Grant No. 201613892119, 201713892009) and Hai Nan Student Innovation and Entrepreneurship Training Program (Grant No. 20160257, 20160202).

\section{REFERENCES}

[1] J. R. Zhang, M. Wang, G. Y. Zhao, Proficient in Labview virtual instrument programming and case realization, Beijing: People Post Press , 2013, pp. 362-368.

[2] J. H. Liu, Virtual instrument design based on LabVIEW, Beijing: Electronic Industry Press, 2003, pp. 58-67.

[3] G. S. Chen, Y. K. Guo, Z. L. Wang, Proficient in Labview programming, Beijing: Electronic Industry Press, 2005, pp. 16-34.

[4] S. Josifovska, The father of LabVIEW, IEEE Review,2003,49(9):30-33.

[5] K. R. Aaron, N. L. Foster, D. P. Hazel, A. M. Basher, Closed-loop position control system using LabVIEW, SoutheastCon., 2002, 12(9): 259 262.

[6] Q. S. Zhang, LED application circuit selection, Beijing: Machinery Industry Press, 2009, 65-69. 